\title{
Coordinated Cluster/Double Star observations of dayside reconnection signatures
}

\author{
M. W. Dunlop ${ }^{1,2}$, M. G. G. T. Taylor ${ }^{3}$, J. A. Davies ${ }^{1}$, C. J. Owen ${ }^{3}$, F. Pitout ${ }^{4}$, A. N. Fazakerley ${ }^{3}$, Z. Pu ${ }^{5}$, H. Laakso ${ }^{4}$,

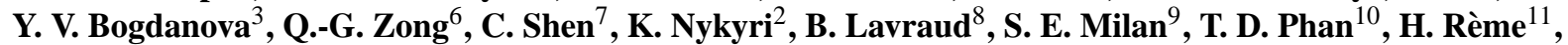

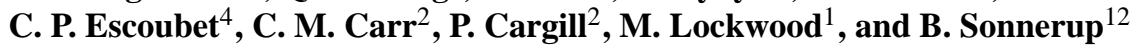 \\ ${ }^{1}$ Space Science and Technology Department, Rutherford Appleton Laboratory, Chilton, Oxfordshire, OX11 0QX, UK \\ ${ }^{2}$ The Blackett Laboratory, Imperial College London, London, SW7 2AZ, UK \\ ${ }^{3}$ Mullard Space Science Laboratory, University College London, Dorking, Surrey, RH5 6NT, UK \\ ${ }^{4}$ ESA/ESTEC, Keplerlaan 1, 2200 AG Noordwijk, The Netherlands \\ ${ }^{5}$ School of Earth and Space Sciences, Peking University, Beijing 100871, China \\ ${ }^{6}$ Centre for Space Physics, Boston University, Boston, Massachusetts, MA 02215, USA \\ ${ }^{7}$ Centre for Space Science and Applied Research, Chinese Academy of Sciences, Beijing 100080, China \\ ${ }^{8}$ Space Science and Applications, Los Alamos National Laboratory, Los Alamos, NM 87545, USA \\ ${ }^{9}$ Department of Physics and Astronomy, University of Leicester, Leicester, LE1 7RH, UK \\ ${ }^{10}$ Space Sciences Laboratory, University of California, Berkeley, California, CA 94720, USA \\ ${ }^{11}$ Centre d'Etude Spatiale des Rayonnements, Toulouse Cedex 4, France \\ ${ }^{12}$ Thayer School of Engineering, Dartmouth College, Hanover, New Hampshire, NH 03755, USA
}

Received: 17 February 2005 - Revised: 9 May 2005 - Accepted: 8 April 2005 - Published: 8 November 2005

Part of Special Issue "Double Star - First Results"

\begin{abstract}
The recent launch of the equatorial spacecraft of the Double Star mission, TC-1, has provided an unprecedented opportunity to monitor the southern hemisphere dayside magnetopause boundary layer in conjunction with northern hemisphere observations by the quartet of Cluster spacecraft. We present first results of one such situation where, on 6 April 2004, both Cluster and the Double Star TC-1 spacecraft were on outbound transits through the dawnside magnetosphere. The observations are consistent with ongoing reconnection on the dayside magnetopause, resulting in a series of flux transfer events (FTEs) seen both at Cluster and TC-1, which appear to lie north and south of the reconnection line, respectively. In fact, the observed polarity and motion of each FTE signature advocates the existence of an active reconnection region consistently located between the positions of Cluster and TC-1, with Cluster observing northward moving FTEs with $+/-$ polarity, whereas TC- 1 sees $-/+$ polarity FTEs. This assertion is further supported by the application of a model designed to track flux tube motion for the prevailing interplanetary conditions. The results from this model show, in addition, that the low-latitude FTE dynamics are sensitive to changes in convected upstream conditions. In particular, changing the interplanetary magnetic field (IMF) clock angle in the model suggests that TC-1 should miss the resulting FTEs more often than Cluster and this is borne out by the observations.
\end{abstract}

Correspondence to: M. W. Dunlop

(m.w.dunlop@rl.ac.uk)
Keywords. Space plasma physics (Magnetic reconnection) - Magnetospheric physics (Magnetopause, cusp, arid boundary layers) - Radioscience (Instruments and techniques)

\section{Introduction}

Reconnection of the Earth's dayside magnetic field with the interplanetary magnetic field (IMF) readily facilitates the transfer of momentum and energy from the solar wind into the Earth's magnetosphere. The process of plasma penetration through the magnetopause via reconnection was first discussed by Dungey (1961), assuming a purely southwarddirected IMF field which presents the optimal conditions for reconnection in the subsolar region. Different IMF orientation and solar wind conditions give rise to varying rates of reconnection (Smith and Lockwood, 1996) as well as variations in the location of the reconnection site (e.g. Crooker, 1979; Gosling et al., 1991; Kessel et al., 1996). The morphology and dynamics of this momentum and energy transfer is still a very active area of space plasma research, in particular the nature of flux transfer events (FTEs) (Russell and Elphic, 1978). FTEs are considered to be the signatures of transient or bursty reconnection, with newly reconnected flux at the subsolar region convecting tailward in the form of a tube-like structure threading the magnetopause (Russell and Elphic, 1978, 1979; Smith and Lockwood, 1996; Rijnbeek et al., 1982, 1984). FTEs were originally characterised 

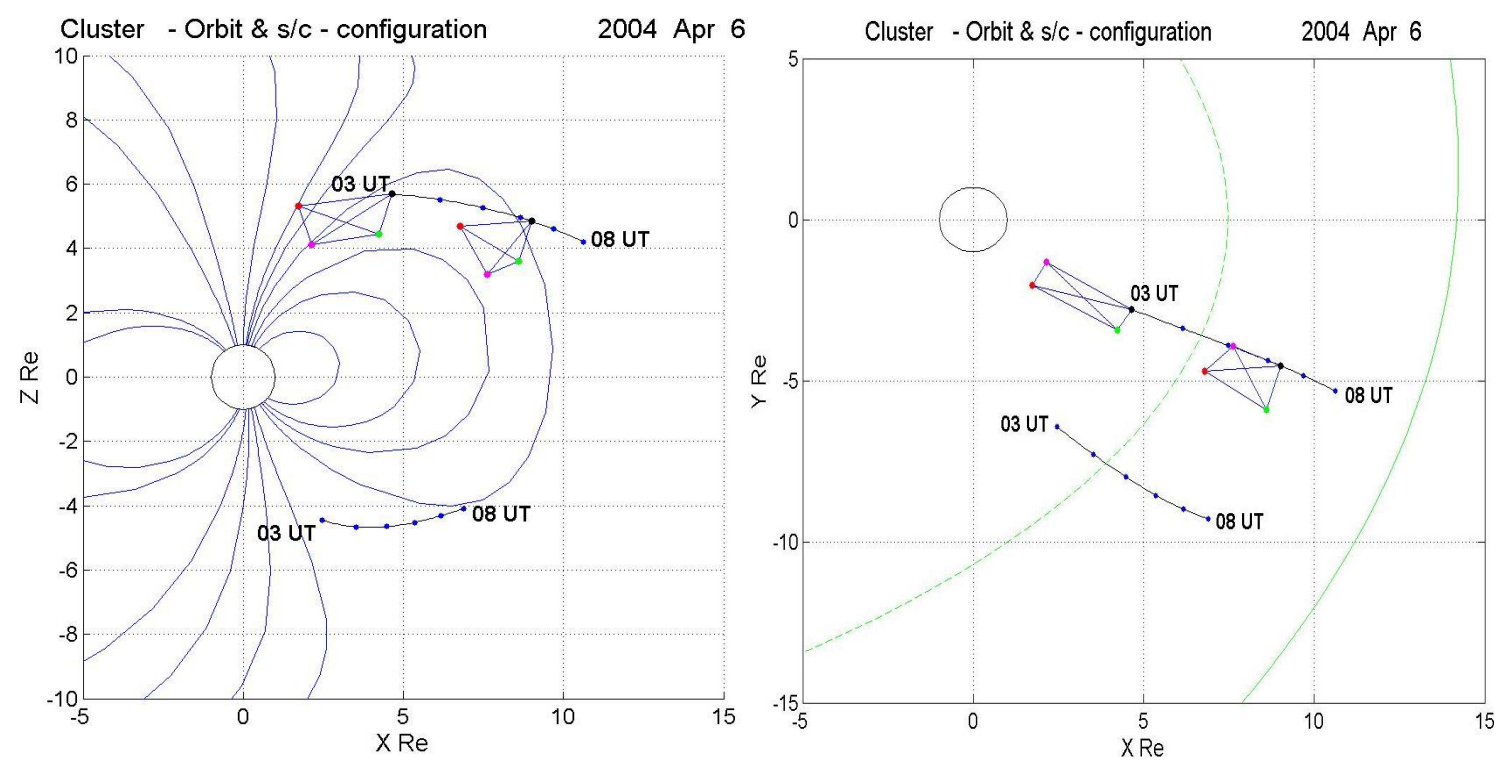

Fig. 1. Cluster s/c1 and Double Star TC-1 tracks in GSM coordinates for the interval 03:00 to 08:00 UT on 6 April 2004. The Cluster orbit also shows two spacecraft configurations (scaled up by a factor x50). Each orbit has hour markers. Model field lines are shown for the projection into the $\mathrm{X}, \mathrm{Z}$ plane and cuts through the bow shock and magnetopause are shown for the $\mathrm{X}, \mathrm{Y}$ plane. For the $\mathrm{X}, \mathrm{Z}$ plane field lines are drawn from the Tsyganenko ' 89 model for guidance.

according to their bipolar oscillation in the magnetic field component normal to the magnetopause, with subsequent studies (e.g. Daly et al., 1981; Thomsen et al., 1987) detailing the intricate mixing of magnetosheath and magnetospheric plasma populations associated with these signatures. In addition, FTE signatures have also been attributed to the effect of solar wind pressure pulses, inducing large-amplitude magnetopause waves (e.g. Sibeck et al., 1989), although the apparent correlation of their occurrence with periods of southwarddirected magnetic field (e.g. Rijnbeek et al., 1984; Lockwood, 1991) favours the reconnection interpretation.

In the context of the Russell and Elphic model, the polarity of the magnetic field signature of an FTE can be used as an indication of which hemisphere the flux tube is connected to (e.g. Rijnbeek et al., 1982; Berchem and Russell, 1984), at least sufficiently near the subsolar region, where the magnetosheath flow is sub-Alfvenic. It is also expected that these events will have corresponding signatures in the high-altitude cusp region, as an extension of the low latitude boundary layer (LLBL), although the characteristic signatures could differ from dayside exterior boundary layer observations (Lockwood et al., 2001). Recent high-latitude, in situ measurements (Lockwood et al., 2001; Owen et al., 2001) by the four spacecraft of the European Space Agency's Cluster mission (Escoubet et al., 2001) have provided extremely detailed and revealing multi-point measurements of the high-latitude magnetopause. Because of the often sporadic nature of the interaction of the solar wind with the magnetosphere, simultaneous coverage over a range of different magnetopause sites, previously only available through fortuitous spacecraft conjunctions (e.g. Wild et al., 2005), provides key information not available with single point mea- surements. The recent launch of the Double Star TC-1 spacecraft into an equatorial orbit provides a unique opportunity to investigate the dayside magnetopause region simultaneously at northern (Cluster) and southern (TC-1) latitudes. In this paper we present preliminary results of the analysis of a Double Star/Cluster conjunction, investigating the evolution of FTEs across the dayside magnetopause. We put our results in context by comparing them to a model of flux tube motion across the magnetopause (Cooling et al., 2001) to ascertain limits on the size and location of the expected reconnection site.

\section{Instrumentation/experimental arrangement}

The Cluster spacecraft (Escoubet et al., 2001) were launched in pairs in July and August 2000 into a polar orbit, with an orbital period of $57 \mathrm{~h}$ and with a perigee and apogee of 4 and 19.6 Earth radii $\left(\mathrm{R}_{E}\right)$, respectively. Since the orbital plane of Cluster is fixed in the inertial frame of the Earth, apogee precesses through $24 \mathrm{~h}$ of Local Time (LT) with a 12-month periodicity. In April 2004, apogee was in the pre-noon sector, near 10:00 LT. In this paper we compare observations from Cluster with those from the first of the pair of Double Star spacecraft, TC-1 (Liu et al., 2005, this issue). The TC-1 spacecraft was launched in December 2003 into an equatorial orbit at $28.2^{\circ}$ inclination, with an orbital period of $27.4 \mathrm{~h}$, a perigee altitude of $570 \mathrm{~km}$ and an apogee of $13.4 \mathrm{R}_{E}$. Figure 1 presents the tracks of both the Cluster and TC- 1 spacecraft for the interval that extends from 03:00 to 08:00 UT on 6 April 2004, in the X-Z (left hand panel) and X-Y (right hand panel) planes, in the Geocentric Solar Magnetic (GSM) 
coordinate system. Also shown is the configuration of the Cluster spacecraft array, at two points along the orbit; the inter-spacecraft separations were a few hundred kilometres during this pass. The interval corresponds to an outbound magnetopause traversal by Cluster, which crosses through the dayside magnetosphere to exit into the magnetosheath at high northern latitudes as shown (note that the actual magnetopause crossing occurred at $\sim 04: 30 \mathrm{UT}$ at Cluster). The plot also shows that TC-1 was also outbound and passed through the magnetopause in the pre-noon sector, dawnwards of Cluster. The TC-1 spacecraft, however, was located in the southern hemisphere. It happened that both the four Cluster spacecraft and TC-1 exited the magnetopause within half an hour of each other.

We concentrate, in this preliminary study, on data from the magnetic field and thermal plasma instruments on Cluster and TC-1. This is facilitated by common instrumentation on the two missions. The four Cluster spacecraft and in fact both Double Star satellites carry FluxGate Magnetometers (FGM). Each FGM instrument comprises a pair of fluxgate magnetic field sensors mounted on an axial boom, although Double Star uses a sensor design different to that used on Cluster (for descriptions of each, see Balogh et al., 2001 and Carr et al., 2005, this issue). The PEACE - Plasma Electron And Current Experiment - instrument on Cluster, as discussed by Johnstone et al. (1997), comprises two separate electron sensors, LEEA (Low-Energy Electron Analyzer) and HEEA (High-Energy Electron Analyzer). The payload of Double Star TC-1 includes the Cluster flight spare of the PEACE/LEEA sensor whilst the spare PEACE/HEEA sensor is carried on the polar Double Star TC-2 spacecraft (Fazakerley et al., 2005, this issue). Similarly, whilst the CIS - Cluster Ion Spectrometry (Rème et al., 2001) - experiment onboard Cluster comprises both CODIF (COmposition DIstribution Function) and HIA (Hot Ion Analyser) components, TC-1 carries only the HIA instrument (Rème et al., 2005, this issue), which provides three-dimensional distributions of the ions which are assumed to be protons.

\section{Results}

\subsection{Solar wind conditions}

During the interval of interest, that extended from 03:00 to 06:00 UT on 6 April 2004, the IMF (when lagged to the Earth), as diagnosed by the MAG experiment (Smith et al., 1998) on the ACE spacecraft (Stone et al., 1998), was predominantly southward $\left(\mathrm{B}_{Z}\right.$ negative) and exclusively dawnward $\left(\mathrm{B}_{Y}\right.$ negative). For most of this interval, $\mathrm{B}_{Z}$, was around $-5 \mathrm{nT}$ (in GSM coordinates) and the $\mathrm{B}_{Y}$ component varied between -8 and $-4 \mathrm{nT}$, so that the IMF clock angle (see bottom panel of Fig. 2) varied. We highlight here that during the interval 04:00-05:40 UT (lagged time), the clock angle first decreased from around -100 to $-150 \mathrm{deg}$. at 05:00 UT and subsequently increased back to $\sim-100 \mathrm{deg}$. The solar wind density, from the ACE/SWEPAM instrument
(McComas et al., 1998) reduced from 6 to $3 \mathrm{~cm}^{-3}$ through the interval, whilst the solar wind velocity varied between 500 and $560 \mathrm{~km} / \mathrm{s}$, resulting in a prevailing solar wind dynamic pressure of $\sim 3-2 \mathrm{nPa}$. The existing IMF conditions were conducive to dayside low-latitude (subsolar) reconnection (see, for example, Moore et al., 2002).

\subsection{Cluster/Double Star observations}

Data from all four Cluster spacecraft and for the Double Star TC-1 spacecraft, for the interval under study where both spacecraft underwent outbound traversals from the magnetosphere into the magnetosheath, are summarised in Fig. 2. Cluster crosses the magnetopause at high northern latitudes and dawnward of local noon at about 10:00 LT and TC-1 crosses the magnetopause at high southerly latitudes, further dawnward at $\sim$ 08:00 LT. The first and second panels of Fig. 2 present spectrograms of spin-averaged, differential electron energy flux from the HEEA sensor of PEACE on the Cluster spacecraft 3 and TC-1, respectively. The third and fourth panels present differential ion energy flux from the HIA sensor for the same two spacecraft. The lower panels show magnetic field data from FGM on all Cluster spacecraft and on TC-1, with the lagged, IMF clock angle at the bottom. A number of distinct features within the interval are immediately apparent. Exits into the magnetosheath are clear both in the plasma and magnetic field data, and indicate magnetopause crossings at 04:15 UT for TC-1 and 04:33 UT for Cluster (the latter indicated by the large magnetic shear at 04:33 UT). Due to the southerly and dawnward location of the TC- 1 spacecraft the $\mathrm{X}$ and $\mathrm{Y}$ components of the magnetospheric field are reversed compared to Cluster. In addition, some significant draping of the magnetosheath field between the spacecraft locations is apparent. This effect is revealed in the different value of the $\mathrm{X}_{\mathrm{GSM}}$ component which is negative at TC-1 and positive at Cluster. Both of these factors result in a much lower local magnetic shear across the magnetopause at TC-1 so that the magnetic field signature of the magnetopause crossing at TC- 1 is less clear than at Cluster. The plasma data from TC- 1 shows a number of partial crossings of the boundary layer before final entry into the magnetosheath.

Superimposed on the underlying time series signatures of both Cluster and TC-1, in both the magnetosphere and magnetosheath, are a number of transient, mixed plasma signatures characteristic of FTEs. For the interval near the magnetopause crossings ( $\sim 04: 10-04: 35$ UT), both magnetosheath and magnetospheric FTE signatures are seen at each spacecraft location. Since there is a low magnetic shear across the magnetopause at TC- 1 , the observation of FTEs at this spacecraft suggests that these signatures are not locally generated, but arise from a (possibly common) distant reconnection site. Furthermore, it is apparent that, in the interval between 04:30 UT and 05:30 UT when both spacecraft are in the magnetosheath, there are significantly more and better defined FTEs observed at the Cluster spacecraft than at TC1. We investigate further below the degree to which the FTE 


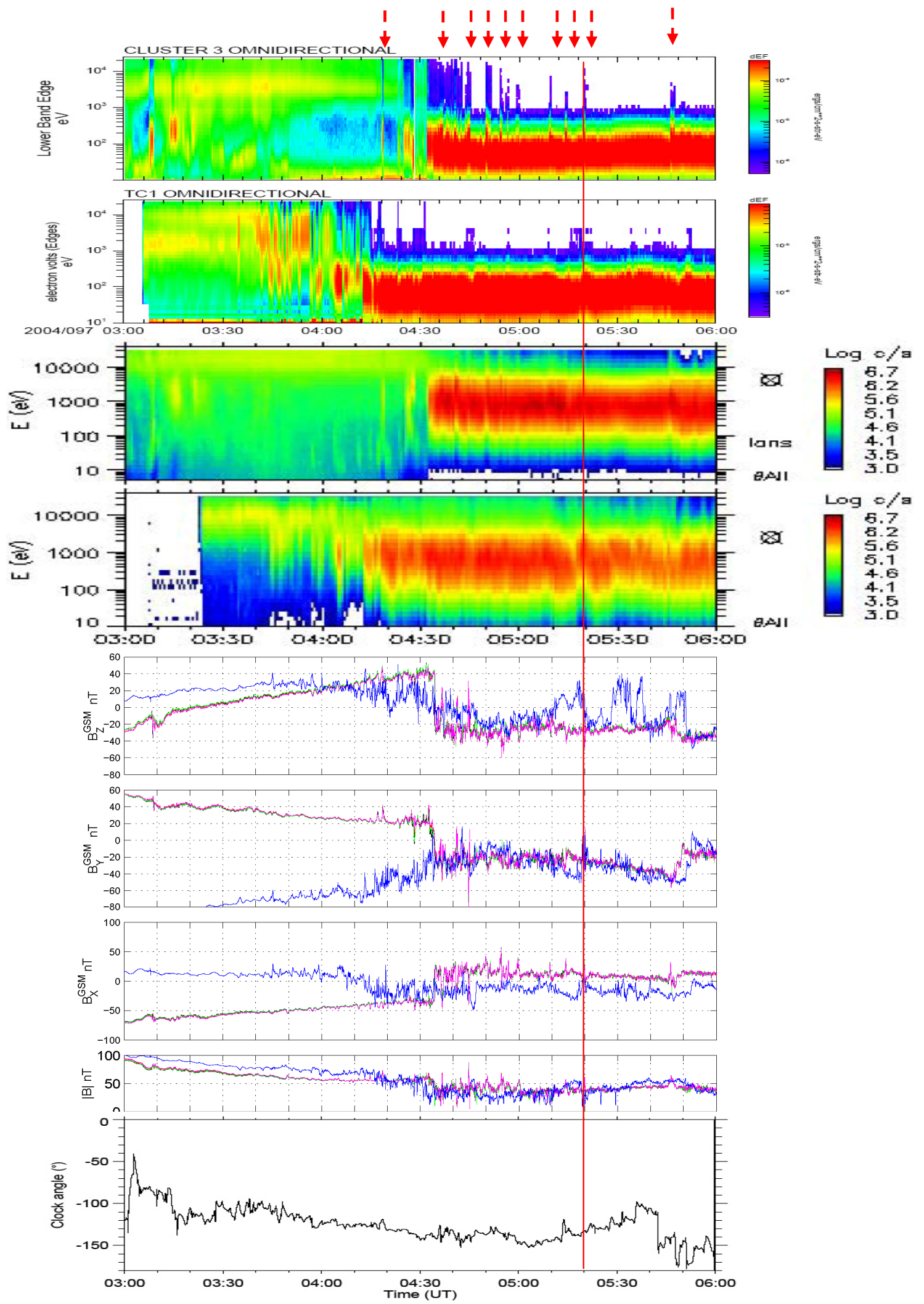

Fig. 2. Summary of the PEACE, HIA, and FGM measurements for the interval shown. The plots for PEACE and HIA are in the same format for Cluster 3 and TC-1, respectively, in both cases and show spin and pitch angle averaged, differential energy flux. The FGM plots show data from all four cluster spacecraft (1-black, 2-red, 3-green, 4-magenta) and TC-1 (in blue). A number of the FTE signatures are indicated by arrows at the top of the plot. The FTE discussed in the text is indicated also by the vertical red line (timed at Cluster). The lagged, IMF clock angle, obtained from ACE data, is shown in the bottom panel. 


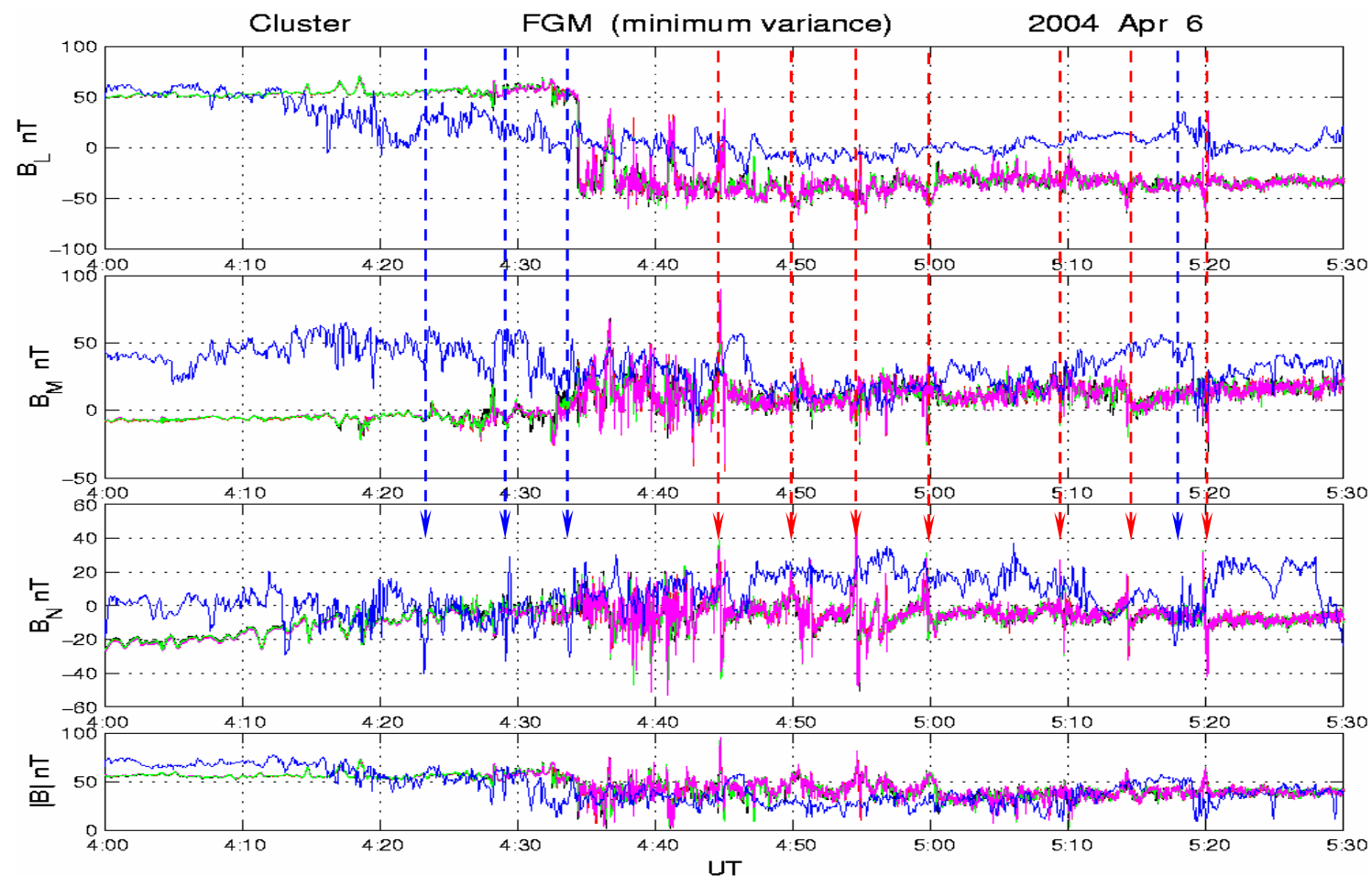

Fig. 3. A multi spacecraft plot of the magnetic field in LMN (MVA) coordinates. The analysis of Cluster gives: [ $\mathrm{n}=0.7200 .1630 .675$, $\mathrm{m}=-0.379-0.7220 .579, \mathrm{l}=-0.5820 .6720 .458], \lambda=5$ and TC- 1 gives $[\mathrm{n}=0.233-0.682-0.694, \mathrm{~m}=-0.679-0.6250 .385,1=0.696-0.381$ 0.609] , $\lambda=3$ (components in GSM). Clear FTEs are observed at Cluster (all spacecraft) with $+/-$ polarity. The FTEs at TC-1 are less clear, but most have $-/+$ (reverse) polarity.

occurrence and behaviour are as a result of the respective spacecraft locations and observed changes in IMF clock angle. Indeed, for a number of these signatures, FTEs are found to occur within 1-2 min of each other at Cluster and TC-1, both on the magnetosheath and magnetospheric sides of the magnetopause and therefore possibly arise from a common merging point (see the model comparison below). One such common FTE, discussed below, is indicated by the vertical red line in Fig. 2.

In order to show these FTE signatures more clearly, Fig. 3 presents magnetic field components in minimum variance (MVA) coordinates (Sonnerup and Cahill, 1967) for Cluster and TC-1 from 04:00 to 05:30 UT on 6 April 2004. The analysis is performed independently on the magnetic field data from the Cluster and TC-1 spacecraft for a short ( $\sim 4$ min) interval around the main magnetopause crossing. The ordering of the interval shown is clearly much better in the case of Cluster than for TC-1, which is as a result of the less well defined magnetopause crossing (in the magnetic field) in the case of TC-1. We refer to these MVA coordinates as LMN, since for both Cluster and TC-1, the intermediate and maximum eigenvectors lie closely parallel $\left(<5^{\circ}\right)$ to the LM coordinates in the system of Russell and Elphic (1978), defined such that $\mathrm{N}$ is in the outward, magnetopause boundary normal direction, $\mathrm{L}$ lies in the boundary and points north (such that the L-N plane contains the GSM Z-axis), and M also lies in the boundary, pointing west. The clearest FTEs in the data from both spacecraft are identified by the dashed, vertical arrows (red for Cluster and blue for TC-1). The last pair of these corresponds to the FTE already mentioned and indicated (for Cluster) by the vertical line in Fig. 2. All FTEs marked on Fig. 3 are listed in Table 1.

In the case of Cluster, all of the FTEs indicated in Fig. 3 have been analysed to determine their orientation and motion and the results of this analysis is briefly summarised in Table 1. The four Cluster spacecraft provide timing information that easily verifies (see, for example, the techniques in Dunlop et al., 2002) that all FTEs at Cluster are moving consistently northwards and each one with different $\mathrm{X}$ and $\mathrm{Y}$ motions, depending on the time of the FTE. The FTE speeds range from $\sim 170 \mathrm{~km} / \mathrm{s}$ to $\sim 250 \mathrm{~km} / \mathrm{s}$. The observed motion of the FTEs changes from eastward $\left(+\mathrm{Y}_{\mathrm{GSM}}\right)$ to westward $\left(-\mathrm{Y}_{\mathrm{GSM}}\right)$ as we move through the magnetosheath interval, and this is related to the change in IMF clock angle during this interval. All FTEs observed at Cluster show standard $+/-$ polarity (as can be observed in Fig. 3), consistent with a draped flux tube signature moving predominantly northward. Conversely, the signatures at TC-1 are much less clear and are fewer in number during the same magnetosheath interval. Moreover, where it can be ascertained, the FTEs at TC-1 show -/+ (reverse) polarity, consistent with a location southward of a reconnection line.

These observations suggest that quasi-steady, or sporadic reconnection is ongoing somewhere between the Cluster and 

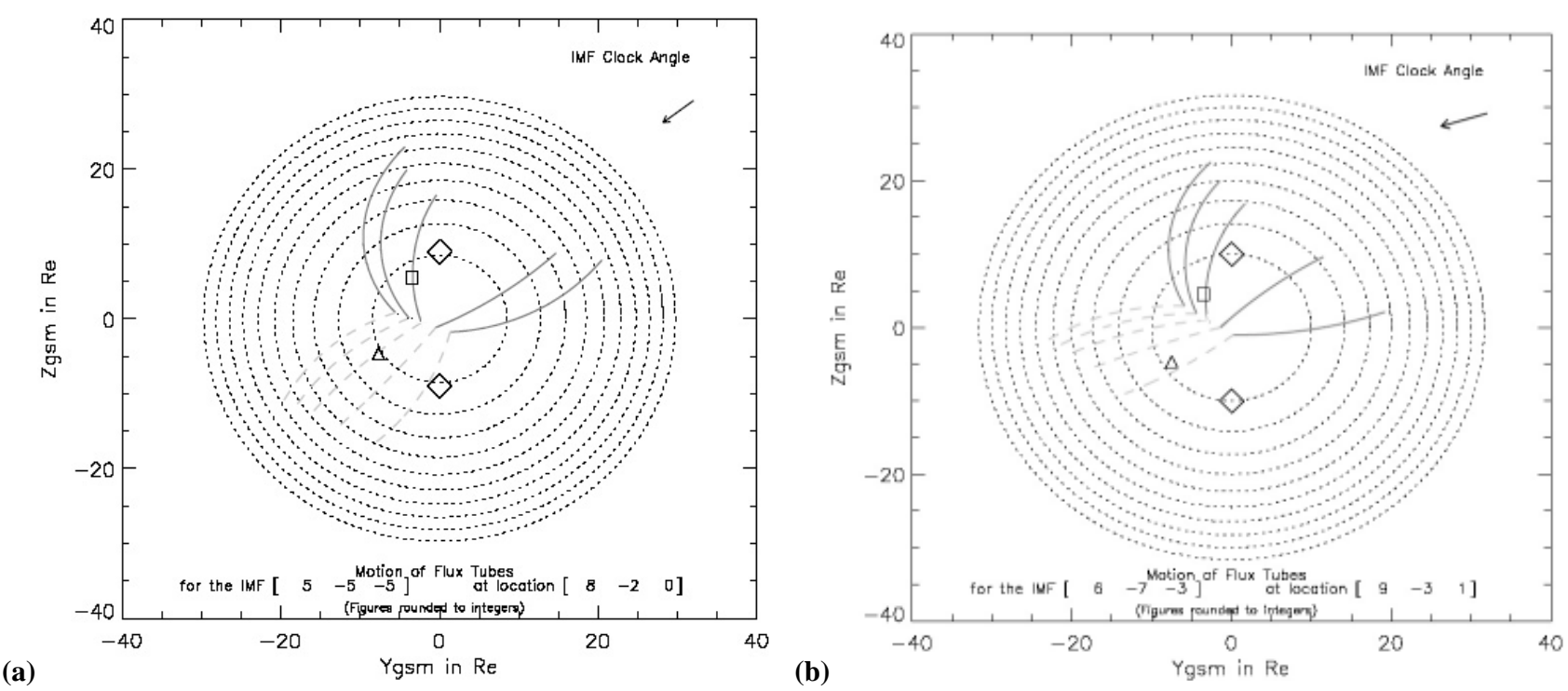

Fig. 4. Results from the Cooling model. The figure is projected in the YZ plane, looking earthward from the Sun. The concentric dotted circles represent the radius of the magnetopause at $5 \mathrm{R}_{E}$ intervals along the $\mathrm{X}$ direction, with the innermost circle representing $\mathrm{X}=5 \mathrm{R}_{E}$. The diamonds represent the position of the cusps for a magnetopause standoff distance of $9 \mathrm{R}_{E}$. The triangle represents the position of Double Star and the square the position of Cluster at the time of the FTE in question. Pairs of open reconnected flux tubes are initiated along the merging line (dot-dashed), with the motion of each tube calculated for a total of $500 \mathrm{~s}$, which is represented by the extent of the line, with the solid line representing flux tubes connected to the northern cusp, and the dashed lines connected to the southern cusp. The IMF orientation is indicated by an arrow in the upper right hand of the figure and the components are stated at the bottom of the plot. The location information pertains to the mid point of the merging line. Other parameters are discussed in the text. Panel (a) shows the results for parameters representing the FTE signature seen at Cluster 05:20 UT and at TC-1 at 05:18:50 UT. Panel (b) shows the effect of modifying the IMF clock angle which moves the region of FTE evolution such that one can envisage Double Star to move out of this region under certain clock angle values.

Table 1. Catalogue of Cluster FTE motions for the FTEs marked on Fig. 3. The normal, n, is obtained directly from timing analysis and represents the direction of the velocity, V. Polarities are marked for each spacecraft. These were not clearly resolved for four of the TC-1 FTEs.

\begin{tabular}{cccccc}
\hline UT_Cluster & Polarity & $\mathrm{n}_{\mathrm{GSE}}$ (motion) & $|\mathrm{V}|$ & UT_TC-1 & Polarity \\
\hline 04:18:00 & & & & $04: 19: 00$ & \\
04:23:00 & & & & $04: 23: 00$ & $-/+$ \\
04:32:00 & & & & $04: 31: 00$ & $-/+$ \\
$04: 37: 00$ & & & $04: 37: 00$ & $-/+$ \\
$04: 45: 00$ & $+/-$ & $-0.9,0.1,0.2$ & 190 & $04: 46: 00$ & \\
$04: 50: 00$ & & & & $04: 48: 00$ & \\
$04: 54: 00$ & $+/-$ & $-0.8,0.4,0.4$ & 250 & $04: 53: 00$ & \\
$04: 56: 00$ & $+/-$ & $-0.8,0.5,0.4$ & 110 & & \\
$05: 00: 00$ & $+/-$ & $0.2,-0.2,0.9$ & 170 & & \\
$05: 09: 00$ & $+/-$ & $-0.4,-0.5,0.7$ & 210 & & \\
$05: 14: 00$ & $+/-$ & $0.2,-0.7,0.7$ & 160 & & \\
$05: 20: 00$ & $+/-$ & $-0.5,-0.5,0.7$ & 230 & $05: 18: 50$ & $-/+$ \\
\hline
\end{tabular}

Double Star spacecraft locations, such that Cluster is better located to observe any resultant FTEs. The motion of the flux tubes, although consistently northward at Cluster, appears to be sensitive to prevailing conditions (the changing IMF clock angle) and precise spacecraft locations. Furthermore, it is possible that some nearly coincident signatures arise from a common reconnection onset, which would send north and south branches of reconnected flux to each of the Cluster and TC-1 locations, respectively. These features, and whether they arise from the establishment of multiple or a common $\mathrm{X}$-line, can be tested to some degree using the model of flux tube motion discussed below.

\subsection{Model comparison}

We have employed the model of Cooling et al. (2001) to study the motion of newly reconnected field lines across the dayside magnetopause. This model initially determines, for given IMF conditions and known magnetopause position, the draping and strength of the magnetosheath field and the flow velocity and density over the entire surface of a paraboloid magnetopause, so setting up a test for a reconnection geometry. This model was a development from that of Cowley and Owen (1989), in which a planar approximation to the magnetopause was adopted. If, for a given location on the magnetopause, the applied condition for steady state reconnection between the magnetosheath and modelled magnetopause is satisfied, the subsequent motion of the newly reconnected field lines across the magnetopause into the magnetotail is traced. The corresponding reconnection $\mathrm{X}$-line of predefined length (taken here to be $5 \mathrm{R}_{E}$ ), centred at a chosen location, 
is constructed in the direction of the merging current calculated at the reconnection site. We do not detail the model here, except by noting these inputs to the model (taken from the known local conditions). The output of the model is summarised by Fig. 4 and discussed below. In general, the selection of the reconnection point implicitly accepts component reconnection as a viable possibility but the model can use the magnetic shear and corresponding magnetopause current to suggest the most likely X-line location, which would generally be the position corresponding to the anti-parallel reconnection condition.

We have run the Cooling model initially commensurate with conditions in the solar wind just before 05:00 UT on 6 April 2004, to be optimum for an FTE signature seen at Cluster at $\sim 05: 20$ UT and at TC- 1 at $\sim 05: 18: 50$ UT. To this end the model was input with values of the $\mathrm{IMF}-\mathrm{B}=(5$, $-5,-5)_{\mathrm{GSE}}$, a solar wind density of $\sim 6 \mathrm{~cm}^{-3}$, a solar wind velocity of $\sim 520 \mathrm{~km} / \mathrm{s}$, and a fitted magnetopause position (to match the magnetopause crossing locations at TC-1 and Cluster) of $\sim 9 \mathrm{R}_{E}$ at the subsolar point. The results are shown in Fig. 4a, which shows a map on the dayside hemisphere. The cusp positions are shown by the diamonds and the set of solid and dashed lines are tracks of the motion of each flux tube northward and southward of the reconnection line, respectively. The set of flux tube tracks deeply engulf both spacecraft and their geometry suggests that oppositely directed FTEs, from northward moving and southward moving branches, may well be seen at each spacecraft location (indicated by the square, Cluster, and triangle, TC-1, symbols). Moreover, the tracks at Cluster, emanating from the whole length of the X-line, show a wide spread of Y directions, suggesting that FTEs may be observed with speeds having different $\mathrm{Y}$ components, as is the case. Thus, for this run, the merging line position and flux tube evolution fits well with the direction and timing of the FTE motion observed by Double Star and Cluster. Moreover, for the particular linked pair of tracks passing through the spacecraft positions, the southward branch of the model flux tube arrives at TC- 1 about $70 \mathrm{~s}$ before the one at Cluster: as was the case for the particular pair of FTEs observed at 05:20 UT (Cluster) and $\sim 05: 18: 50$ UT (TC-1). Note that in the model, the velocity is known along the track of each flux tube and each track has a particular, known length, so that the predicted time to arrive at all positions along the length of the track is known and the times of the FTE pair, in particular, can be calculated.

Comparative runs were also made to explore the sensitivity of the results to different clock angles and X-line location. An example of this is shown in Fig. 4b, using the minimum clock angle that occurred during the interval 03:30 to 05:40 UT. In this case, one can see that such modification of the driving conditions could result in the convection flow of reconnected flux tubes turning more dawnward, moving TC1 to the edge of the FTE convection region, and so reducing the number of clear FTEs observed by TC-1 as compared to those observed by Cluster during this crossing. This fits very well with the observation that TC-1 sees few clear FTEs be- tween 04:30 and 05:30 UT. In addition it is evident that the northern pattern of tracks, which shows a spread of Y directions are possible for any FTE motion, is fairly stable to changing conditions, again consistent with Cluster observing FTEs all through the interval, with consistent polarity, but each with different motion in the $\mathrm{Y}_{\mathrm{GSE}}$ direction.

A modified version of the Cooling model, in which the condition for steady-state reconnection was relaxed, was developed by Wild et al. (2005) to aid a comparative study of FTE signatures observed by Cluster and Geotail. This modification enabled the authors to compare the expected flux tube motion with the FTE signatures without constraining the location of the reconnection site using an assumed threshold to the reconnection process. The authors were able to demonstrate that their observations were consistent with the motion of northward (southward) and tailward moving flux tubes anchored in the northern (southern) hemisphere passing in close proximity to the Cluster (Geotail) spacecraft, and infer an approximate position of the reconnection site, which in that case was near-equatorial. In the present study, TC-1 lies further south and therefore further from the X-line studied by Wild et al. (2005), Cluster and TC-1 being nearly equidistant from the X-line. Nevertheless, both studies suggest that a single reconnection site, near the subsolar point, is the very likely explanation of the events.

\section{Conclusions}

In this paper we have presented data during a magnetopause conjunction between Cluster and the Double Star, TC-1 spacecraft, for which both spacecraft are outbound, with Cluster situated north and just dawnward of the sub-solar region and TC-1 situated south and further dawnward of the sub-solar region. The data suggest a period of ongoing reconnection with a common X-line extending over limited LT (modelled at $\sim 5 \mathrm{R}_{E}$ length) located between Cluster and TC1. In particular, a series of FTE signatures are observed at both spacecraft locations with those at Cluster having +/polarity (and a northward motion, confirmed by four spacecraft timing analysis) and those at TC- 1 have $-/+$ polarity (implying a southward motion), consistent with moving flux tubes arising from a single reconnection line. The position of TC-1, which crosses into the magnetosheath earlier than Cluster, is consistent with the FTEs observed at TC- 1 having significant southward and dawnward directions of motion. The Cluster-FTEs (with speeds ranging over $\sim 170$ $250 \mathrm{~km} / \mathrm{s}$ ) move either duskward or dawnward until around 05:00 UT and then move predominantly dawnward. Moreover, the FTE observations by TC-1 are more concentrated around a short time after its exit into the magnetosheath (before Cluster crosses the magnetopause) and subsequently, TC-1 does not see as many or such clear signatures as Cluster until after 05:00 UT. Both this change in the FTE motion at Cluster and the FTE occurrence at TC-1 can be understood since, during the same period, the IMF clock angle is variable, ranging between -120 to $-100 \mathrm{deg}$., until just after 
05:00 UT, subsequently becoming more negative, at around $-140 \mathrm{deg}$.

This interpretation is quantitatively borne out by the application of the Cooling model, which places the X-line near the sub-solar point, but extending dawnward (a result of the strong negative IMF- $\mathrm{B}_{Y}$ ). The precise location of the X-line is selectable in the model and was chosen here to result in a good timing fit for the expected flux tube motion. We note that this selection of the reconnection point implicitly accepts component reconnection as a viable possibility, although the model may be run so as to identify an anti-parallel location. There are periods between Cluster and TC- 1 where common FTE signatures are possible (i.e. the opposite branches of the Cooling model), however. One possible, common signature occurs at 05:20 UT, where TC- 1 sees the signature $\sim 70 \mathrm{~s}$ before Cluster. The model has been run for the particular conditions most relevant to that FTE and results in coincident tracks for the north and south branches of flux tubes, which arrive at each spacecraft location very close to the respective times observed. Overall, the $\mathrm{X}$-line fit agrees well with all the features mentioned above as observed by both Cluster and TC-1. Subsequent runs of the Cooling model were carried out to examine the effect of modified X-line location and clock angle, and suggest that TC- 1 can often miss the convection region of the FTEs and that the dawn-dusk motion, in particular with the case of Cluster, is modified by slight change in the solar wind conditions (as occurs between 04:30-05:30 UT). We note that the sampling by both spacecraft of other common flux tube signatures may depend upon proximity of the spacecraft to the magnetopause and this analysis does not preclude other north/south pairs of reconnected flux tubes being missed more often by TC-1, which exits into the magnetosheath earlier than Cluster.

In summary, we have shown here:

- A close magnetopause conjunction between Cluster and TC-1, dawnward of noon, with Cluster at high latitude and TC-1 south of the sub-solar region, during a period of ongoing reconnection, with an $\mathrm{X}$-line between the spacecraft.

- A series of FTE signatures are observed in both spacecraft that are consistent in polarity and motion with this geometry.

- The Cluster-FTEs move predominantly dawnward at times later than 05:00 UT but some have duskward motion at earlier times.

- TC-1 crosses into the magnetosheath earlier than Cluster and fewer TC-1 FTEs are observed than Cluster FTEs.

- A possible common signature (flux tube branches arising from the same reconnection point) occurs at $\sim 05: 20 \mathrm{UT}$, an interpretation quantitatively born out by the application of the Cooling model.
- Comparative runs of the model confirm changes arising from: modified $\mathrm{X}$-line location and modified clockangle and confirm that TC- 1 can often miss FTEs and that their dawn-dusk motion at Cluster can be modified by slight changes in clock angle.

This preliminary study is part of a wider activity to focus on the opportunities arising from the simultaneous flight of the Cluster and Double Star missions. The current work represents an example of the capability of such mission synergy and further work will address a wider database of such events.

Acknowledgements. This preliminary study was born from an ISSI working group on "Comparative Cluster-Double Star measurements of the Dayside Magnetosphere" and the authors would like to thank ISSI, Berne, Switzerland, for its sponsorship of this group. We also acknowledge C. W. Smith, N. F. Ness and the Bartol Research Institute (BRI), D. J. McComas, R. Skoug and the Los Alamos National Laboratory for use of the level 2 ACE MAG and ACE Solar Wind Experiment data, respectively. This work is also supported by the CNSF Grant 40390150 and Chinese Fundamental Research Project G200000784.

Edited by: T. Pulkkinen

Reviewed by: J. A. Wild and another referee

\section{References}

Balogh, A., Carr, C. M., Acuna, M. H., Dunlop, M. W., Beek, T. J., Brown, P., Fornacon, K.-H., Georgescu, E., Glassmeier, K. H., Harris, J., Musmann, G., Oddy, T., and Schwingenschuh, K.: The Cluster magnetic field investigation: overview of in-flight performance and initial results, Ann. Geophys., 19, 1207-1217, 2001,

SRef-ID: 1432-0576/ag/2001-19-1207.

Berchem, J. and Russell, C. T.: Flux transfer events on the magnetopause: spatial distribution and controlling factors, J. Geophys. Res., 89, 6689-6703, 1984.

Cooling, B. M. A., Owen, C. J., and Schwartz, S. J.: Role of magnetosheath flow in determining the motion of open flux tubes, J. Geophys. Res., 106, 18 763-18 775, 2001.

Cowley, S. W. H. and Owen, C. J.: A simple illustrative model of open flux tube motion over the dayside magnetopause, Planet. Space Sci., 37, 1461-1475, 1989.

Crooker, N. U.: Dayside merging and cusp geometry, J. Geophys. Res., 84, 951, 1979.

Daly, P. W., Williams, D. J., Russell, C. T., and Keppler, E.: Particle signature of magnetic flux transfer events at the magnetopause, J. Geophys. Res., 86, 1628-1632, 1981.

Dungey, J. W.: Interplanetary magnetic field and the auroral zones, Phys. Rev. Lett., 6, 47-48, 1961.

Dunlop, M. W., Balogh, A., Glassmeier, K.-H., et al.: Four-Point Cluster Application Of Magnetic Field Analysis Tools: The Discontinuity Analyser, J. Geophys. Res., 107, 1385, 2002.

Escoubet, C. P., Fehringer, M., and Goldstein, M.: Introduction: the Cluster mission, Ann. Geophys., 19, 1197-1200, 2001,

SRef-ID: 1432-0576/ag/2001-19-1197.

Fazakerley, A. N., Carter, P. J., Watson, G., et al.: The Double Star plasma electron and current experiment, Ann. Geophys., 23, 2733-2756, 2005. 
Gosling, J. T., Thomsen, M. F., Bame, S. J., Elphic, R. C., and Russell, C. T.: Observations of reconnection of interplanetary and lobe magnetic field lines at the high-latitude magnetopause, J. Geophys. Res., 96, 14 097-14 106, 1991.

Johnstone, A. D., Burge, S., Carter, P. J., Coates, A. J., Coker, A. J., Fazakerley, A. N., Grande, M., Gowan, R. A., Gurgiolo, C., Hancock, B. K., Narheim, B., Preece, A., Sheather, P. H., Winningham, J. D., and Woodliffe, R. D.: PEACE: A plasma electron and current experiment, Space Sci. Rev., 79, 351, 1997.

Kessel, R. L., Chen, S.-H., Green, J. L., Fung, S. F., Boardsen, S. A., Tan, L. C., Eastman, T. E., Craven, J. D., and Frank, L. A.: Evidence of high-latitude reconnection during northward IMF: Hawkeye observations, Geophys. Res. Lett., 23, 583-586, 1996.

Liu, Z.-X., Escoubet, C. P., Pu, Z., et al.: The Double Star mission, Ann. Geophys., 23, 2707-2712, 2005.

Lockwood, M.: Flux Transfer Events at the Dayside Magnetopause: Transient Reconnection or Magnetosheath Dynamic Pressure Pulses?, J. Geophys. Res., 96, 5497-5509, 1991.

Lockwood, M., Fazakerley, A., Opgenoorth, H., et al.: Coordinated Cluster and ground-based instrument observations of transient changes in the magnetopause boundary layer during northward IMF, Ann. Geophys., 19, 1641-1654, 2001,

SRef-ID: 1432-0576/ag/2001-19-1641.

McComas, D. J., Bame, S. J., Barker, P., Feldman, W. C., Phillips, J. L., Riley, P., and Griffee, J. W.: Solar wind electron proton alpha monitor (SWEPAM) for the Advanced Composition Explorer, Space Sci. Rev., 86, 561-612, 1998.

Moore, T. E., Fok, M.-C., and Chandler, M. O.: The dayside reconnection X line, J. Geophys. Res., 107(A10), doi:10.1029/2002JA009381, p.SMP 26, 2002.

Owen, C. J., Fazakerley, A. N., Carter, P. J., Coates, A. J., Krauklis, I. C., Szita, S., Taylor, M. G. G. T., Travnicek, P., Watson, G., Wilson, R. J., Balogh, A., and Dunlop, M. W.: Cluster PEACE observations of electrons during magnetospheric flux transfer events, Ann. Geophys., 19, 1509-1522, 2001,

SRef-ID: 1432-0576/ag/2001-19-1509.

Rème, H., Aoustin, C., Bosqued, J. M., Dandouras, I., et al.: First multispacecraft ion measurements in and near the Earth's magnetosphere with the identical Cluster ion spectrometry (CIS) experiment, Ann. Geophys., 19, 1303, 2001,

SRef-ID: 1432-0576/ag/2001-19-1303

Rème, H., Dandouras, I., Aoustin, C., Bosqued, J. M., et al.: The HIA instrument on board Tan Ce 1 Double Star near-equatorial spacecraft and its first results, Ann. Geophys., 23, 2757-2774, 2005.
Rijnbeek, R. P., Cowley, S. W. H., Southwood, D. J., and Russell, C. T.: Observations of reverse polarity flux transfer events at the Earth's dayside magnetopause, Nature, 300, 23-26, 1982.

Rijnbeek, R. P., Cowley, S. W. H., Southwood, D. J., and Russell, C. T.: A survey of dayside flux transfer events observed by ISEE 1 and 2 magnetometers, J. Geophys. Res., 89, 786-800, 1984.

Russell, C. T. and Elphic, R. C.: Initial ISEE magnetometer results: magnetopause observations, Space Sci. Rev., 22, 681-715, 1978.

Russell, C. T. and Elphic, R. C.: ISEE observations of flux transfer events at the dayside magnetopause, Geophys. Res. Lett., 6, 3336, 1979.

Sibeck, D. G., Baumjohann, W., Elphic, R. C., Fairfield, D. H., Fennell, J. F., Gail, W. B., Lanzerotti, L. J., Lopez, R. E., Luehr, H., Lui, A. T. Y., Maclennan, C. G., McEntire, R. W., Potemra, T. A., Rosenburg, T. J., and Takahashi, K.: The Magnetospheric Response to 8-Minute Period Strong-Amplitude Upstream Pressure Variations, J. Geophys. Res., 94, 2505-2519, 1989.

Smith, M. F. and Lockwood, M.: Earth's magnetospheric cusps, Rev. Geophys., 34, 233-260, 1996.

Smith, C. W., L'Heureux, J., Ness, N. F., Acuña, M. H., Burlaga, L. F., and Scheifele, J.: The ACE Magnetic Fields Experiment, Space Sci. Rev., 86, 613-632, 1998.

Sonnerup, B. U. O. and Cahill, L. J.: Magnetopause structure and attitude from Explorer 12 Observations, J. Geophys. Res., 72, 171-183, 1967.

Stone, E. C., Frandsen, A. M., Mewaldt, R. A., Christian, E. R., Margolies, D., Ormes, J. F., and Snow, F.: The Advanced Composition Explorer, Space Sci. Rev., 86, 1-22, 1998.

Thomsen, M. F., Stansberry, J. A., Bame, S. J., Fuselier, S. A., and Gosling, J. T.: Ion and electron velocity distributions within flux transfer events, J. Geophys. Res., 92, 12 127-12 136, 1987.

Wild, J. A., Milan, S. E., Cowley, S. W. H., Bosqued, J. M., Reme, H., Nagai, T., Kokubun, S., Saito, Y., Mukai, T., Davies, J. A., Cooling, B. M. A., Balogh, A., and Daly, P. W.: Simultaneous insitu observations of the signatures of dayside reconnection at the high and low latitude magnetopause, Ann. Geophys., 23, 445460, 2005,

SRef-ID: 1432-0576/ag/2005-23-445. 